\title{
Phi-Bonacci Butterfly Stroke Numbers to Assess Self-Similarity in Elite Swimmers
}

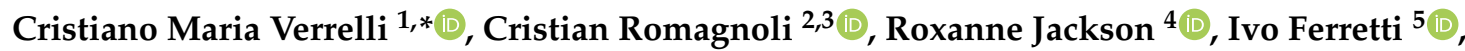 \\ Giuseppe Annino ${ }^{3,6,7}$ (i) and Vincenzo Bonaiuto ${ }^{3}[$
}

1 Department of the Electronic Engineering, University of Rome Tor Vergata, via del Politecnico 1, 00133 Rome, Italy

2 Department for Life Quality Studies, University of Bologna, 47900 Rimini, Italy; cristian.romagnoli2@unibo.it

3 Sport Engineering Lab, Department of Industrial Engineering, University of Rome Tor Vergata, 00133 Rome, Italy; g-annino@hotmail.com (G.A.); vincenzo.bonaiuto@uniroma2.it (V.B.)

4 Institute of Automation Technology, Otto-von-Guericke University Magdeburg, 39106 Magdeburg, Germany; roxanne.jackson@ovgu.de

5 Biomechanical and Video-Analysis Area for the National Teams of Federazione Italiana Nuoto (FIN), 00100 Rome, Italy ; ferrivo@libero.it

6 Department of Medicine Systems, University of Rome Tor Vergata, 00133 Rome, Italy

7 Centro di Biomedicina Spaziale, University of Rome Tor Vergata, 00133 Rome, Italy

* Correspondence: verrelli@ing.uniroma2.it; Tel.: +39-(0)6-72597410

check for updates

Citation: Verrelli, C.M.; Romagnoli, C.; Jackson, R.; Ferretti, I.; Annino, G.; Bonaiuto, V. Phi-Bonacci Butterfly Stroke Numbers to Assess Self-Similarity in Elite Swimmers. Mathematics 2021, 9, 1545. https:// doi.org/10.3390/math9131545

Academic Editor: Isabelle Ramiere

Received: 21 May 2021

Accepted: 22 June 2021

Published: 1 July 2021

Publisher's Note: MDPI stays neutral with regard to jurisdictional claims in published maps and institutional affiliations.

Copyright: (c) 2021 by the authors. Licensee MDPI, Basel, Switzerland. This article is an open access article distributed under the terms and conditions of the Creative Commons Attribution (CC BY) license (https:// creativecommons.org/licenses/by/ $4.0 /)$.

\begin{abstract}
A harmonically self-similar temporal partition, which turns out to be subtly exhibited by elite swimmers at middle distance pace, is formally defined for one of the most technically advanced swimming strokes-the butterfly. This partition relies on the generalized Fibonacci sequence and the golden ratio. Quantitative indices, named $\phi$-bonacci butterfly stroke numbers, are proposed to assess such an aforementioned hidden time-harmonic and self-similar structure. An experimental validation on seven international-level swimmers and two national-level swimmers was included. The results of this paper accordingly extend the previous findings in the literature regarding human walking and running at a comfortable speed and front crawl swimming strokes at a middle/long distance pace.
\end{abstract}

Keywords: generalized Fibonacci sequence; self-similarity; golden ratio; butterfly swimming; elite swimmers

\section{Introduction}

Fibonacci numbers play crucial roles in combinatorial mathematics and elementary number theory. Although such numbers have been investigated for centuries, they continue to intrigue mathematicians and researchers in many areas of human endeavor (as can be seen in [1] and the references therein), while providing new tools for expanding the frontiers of mathematical study. In respect to this, the golden ratio $\phi \approx 1.61803$, namely the positive solution to the equation $x^{2}=1+x$, appears in some very fundamental relationships involving numbers, with one of the most basic occurrences of the golden ratio involving the use of two seed values and a simple Fibonacci-like additive recursion relationship.

Walking and running are human gait modes exhibiting different mechanics and energetics. A double support phase, i.e., both limbs are in ground contact, identifies the walking gait, whereas a double float phase, i.e., no limb is in ground contact, identifies the running gait. However, both physiological (symmetric and recursive) human walking and running are characterized, from a temporal point of view, by only four specific time intervals, associated with the durations of gait cycle, swing, stance and double support (double float) phases. More precisely, physiological symmetric walking (running) is classically recognized to exhibit:

- A stance (swing) duration, $t_{\mathrm{ST}}\left(t_{\mathrm{SW}}\right)$, being close to $62 \%$ of gait cycle duration $t_{\mathrm{GC}}$; 
- A swing (stance) duration being close to $38 \%$ of gait cycle duration;

- A double support (double float) duration, $t_{\mathrm{DS}}\left(t_{\mathrm{DF}}\right)$, being consequently close to $24 \%$ of gait cycle duration.

As recently formally recognized in [2], the above sequence $\{0.24,0.38,0.62,1\}$ can be viewed as a slight approximation of the special sequence in walking:

$$
\Sigma:\left\{t_{\mathrm{DS}} / t_{\mathrm{GC}}, t_{\mathrm{SW}} / t_{\mathrm{GC}}, t_{\mathrm{ST}} / t_{\mathrm{GC}}, 1\right\}=\left\{1 / \phi^{3}, 1 / \phi^{2}, 1 / \phi, 1\right\}
$$

and its conceptual counterpart in running:

$$
\Sigma:\left\{t_{\mathrm{DF}} / t_{\mathrm{GC}}, t_{\mathrm{ST}} / t_{\mathrm{GC}}, t_{\mathrm{SW}} / t_{\mathrm{GC}}, 1\right\}=\left\{1 / \phi^{3}, 1 / \phi^{2}, 1 / \phi, 1\right\} .
$$

The above sequence $\Sigma$ is nothing but a generalized four-length Fibonacci sequence [3] (with the real numbers $1 / \phi^{3}, 1 / \phi^{2}$ as seeds), generally defined, for any values $\gamma$ and $\delta$, by ( $\psi$ is the negative solution to $x^{2}=1+x$ ):

$$
U_{n}=\gamma \varphi^{n}+\delta \psi^{n}
$$

satisfying the recurrence:

$$
U_{n}=\gamma \varphi^{n-1}+\delta \psi^{n-1}+\gamma \varphi^{n-2}+\delta \psi^{n-2}=U_{n-1}+U_{n-2} .
$$

Regarding $\Sigma$ : (i) the general Fibonacci sequence structure is rooted in the following duration constraints of symmetric walking (and running, respectively): $t_{\mathrm{DS}}+t_{\mathrm{SW}}=t_{\mathrm{ST}}$ (respectively, $\left.t_{\mathrm{DF}}+t_{\mathrm{ST}}=t_{\mathrm{SW}}\right), t_{\mathrm{SW}}+t_{\mathrm{ST}}=t_{\mathrm{GC}}$; (ii) the specific $\phi$-dependent (temporally harmonic) self-similar structure relies on the special chain of ratios $t_{\mathrm{SW}} / t_{\mathrm{DS}}=t_{\mathrm{ST}} / t_{\mathrm{SW}}=t_{\mathrm{GC}} / t_{\mathrm{ST}}$ (respectively, $t_{\mathrm{ST}} / t_{\mathrm{DF}}=t_{\mathrm{SW}} / t_{\mathrm{ST}}=t_{\mathrm{GC}} / t_{\mathrm{SW}}$ ) all being equal to $\phi$. Indeed, $1 / \phi^{3} \approx 0.23608$, $1 / \phi^{2} \approx 0.38198,1 / \phi \approx 0.61804$. In other words, conditions in (i)-(ii) apparently induce a repeated self-proportional partition (namely self-similarity), in accordance with the fact that two quantities are in the golden ratio if their ratio is the same as the ratio of their sum to the larger of the two quantities. This way, walking and running implicitly involve a fractal nature, in which the structure of the larger scale resembles the structure of the sub-unit and in which one of the simplest ways of transformation, i.e., the new domain composed of two previous ones, is highlighted.

Very recent experimental and theoretical analyses-inspired by the aforementioned cyclic human movements in walking and running (see also [4,5] — have found harmonic structures to even appear in front crawl swimming [6] at a middle/long distance pace, at which the presence of redundant movements is reduced via in-phase synchronization with the induced waves in the water. With respect to this, the recent paper [6] not only provides a mathematical framework and experimental consistency for recognizing preliminary evidence, for elite front-crawl swimmers swimming at a middle/long distance pace, a recovery phase duration that is very close to the fundamental unit $3 u_{f}$ (where $u_{f}$ is equal to the duration of the front crawl stroke divided by 12) but it also illustrates the existence of harmonic structures-in both their simple and enhanced versions- that elite swimmers seek to reproduce in order to improve their performance. We also refer the reader to $[7,8]$ for a different scenario involving the dimensionless Strouhal number.

A more technically advanced swimming stroke is the butterfly. Simultaneous strokes, such as breaststroke and butterfly, are in fact considered to be highly technical due to the complex coordination of the arm and leg actions [9]. In particular, in butterfly, the out-ofwater arms recovery is facilitated by the leg undulation. To be effective, however, the kick must appear as a consequence of a body wave-like cefalo-caudal undulation motion [10]. Having a glide time with the arms extended forward at the top of the stroke is certainly a strategy used to reduce the energetic cost (metabolic power/velocity) for long-distance swims [11]. This way, the head, trunk and upper limbs are profiled in a streamlined position in order to glide, and consequently, provide some rest at each stroke during a long butterfly 
swim (some aquatic animals such as giant cormorants, penguins, and dolphins improve the metabolic efficiency of swimming by adopting locomotion patterns with alternating periods of propulsion and gliding [10]). However, this is not effective for achieving a higher stroke rate [10] and for avoiding great instantaneous velocity fluctuations. On the other hand, when velocity and stroke rate increase, coordination becomes closer to an in-phase mode [12,13], just like in human locomotion and in quadrupedal coordination. Indeed, the significant skill effect in [10] indicates that elite swimmers-whom are the object of analysis in this paper-have stronger arm/leg synchronization than the sub-elite swimmers: elite swimmers adopt a shorter glide to overcome great forward resistance and generate higher forces during the arm pull; sub-elite swimmers often compensate for coordination mistakes by applying greater force. With respect to this, we also refer the reader to [14] —for devices performing propulsion analysis in swimming; [15]—for a quantitative evaluation of phases of turns during competition; and [16] - for the role of the hip movement in the stroke mechanics.

The aim of this paper was to extend, for the first time in the literature-to the best of our knowledge - the aforementioned findings regarding human walking and running at comfortable speed and front crawl swimming strokes at a middle/long distance pace: a harmonically self-similar temporal partition, which relies on the generalized Fibonacci sequence and the golden ratio, is formally defined for the highly complex and upper and lower-limbs-coordinated butterfly stroke. Quantitative indices, named $\phi$-bonacci butterfly stroke numbers, are accordingly proposed to assess such a hidden time-harmonic and self-similar structure being subtly exhibited by elite swimmers at middle distance pace.

\section{Generalized Fibonacci Sequences in Butterfly Stroke}

This section presents the main result of the paper.

\subsection{Butterfly Stroke Phases}

Different arm and leg phases (see Figure 1) can be identified in a butterfly stroke $S$ with duration $t_{\mathrm{S}}$, here characterized by two leg undulations for one arm stroke $[10,12,17]$. They are in order, for the arms:

(i) Entry and catch phase (between the entry of the hands into the water and the beginning of their backward movement), with duration $t_{\mathrm{EC}}$;

(ii) Pull phase (between the beginning of the backward movement of the hands and their entry into the plane vertical to the shoulders), with duration $t_{\mathrm{PL}}$;

(iii) Push phase (between the positioning of the hands below the shoulders and their exit from the water), with duration $t_{\mathrm{PS}}$;

(iv) Recovery phase (between the arrival of the hands at the water level and their subsequent entry into the water), with duration $t_{\mathrm{RE}}$,

and for the legs:

(i) Downward phase 1 (between the high and low break-even points- at first occurrence-of the feet during the first undulation), with duration $t_{\mathrm{K} 1}$;

(ii) Upward phase 1 (between the low and high break-even points-at first occurrence-of the feet during the first undulation), with duration $t_{\mathrm{U} 1}$;

(iii) Downward phase 2 (between the high and low break-even points-at first occurrence-of the feet during the second undulation), with duration $t_{\mathrm{K} 2}$;

(iv) Upward phase 2 (between the low and high break-even points-at first occurrence-of the feet during the second undulation), with duration $t_{\mathrm{U} 2}$.

The inter-limb coordination of such phases is governed by the time delays (with a positive or negative sign):

- $T_{1}$, between the start of Entry and catch phase and the start of Downward phase 1;

- $T_{2}$, between the start of Pull phase and the start of Upward phase 1;

- $\quad T_{3}$, between the start of Push phase, and the start of Downward phase 2;

- $T_{4}$, between the start of Recovery phase, and the start of Upward phase 2. 
As shown in [10], elite swimmers show a smaller total time gap, taken as the sumexpressed as the percentage of a complete stroke - of the absolute values of $T_{1}, T_{2}, T_{3}, T_{4}$ and used to assess the effectiveness of the global arm-leg coordination.

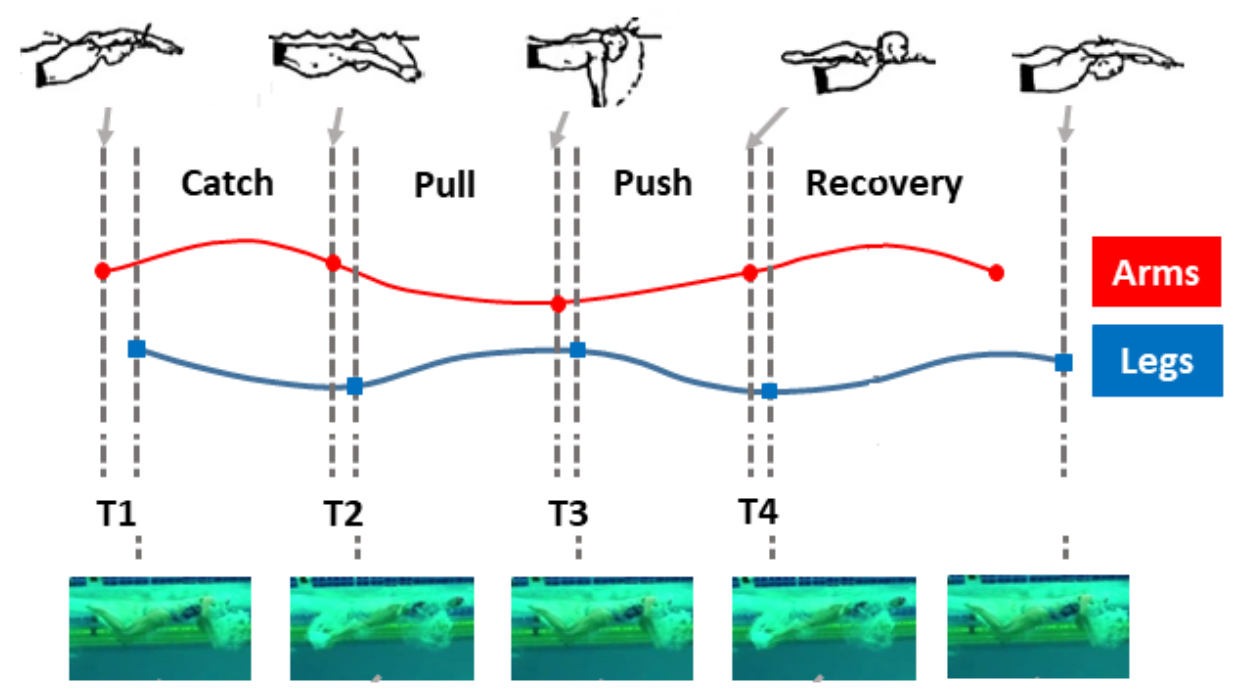

Figure 1. Butterfly stroke: arm and leg phases (case of $T_{2}, T_{3}, T_{4}>0$ ) according to [12].

\subsection{Self-Similarity and Enhanced Self-Similarity in Butterfly Stroke}

We first define the repetitive butterfly stroke, in which $T_{1}$ of the subsequent stroke equals $T_{1}$ of the stroke under investigation, so that the arm stroke (namely from the start of Entry and catch phase to the start of the subsequent Entry and catch phase) equals the leg stroke (namely, from the start of Downward phase 1 to the start of the subsequent Downward phase 1). A repetitive butterfly stroke that is characterized by its values $\left(T_{1} / t_{\mathrm{S}}=a[\%], T_{2} / t_{\mathrm{S}}=b[\%], T_{3} / t_{\mathrm{S}}=c[\%], T_{4} / t_{\mathrm{S}}=d[\%]\right)$ is denoted hereafter by $(a, b, c, d)$-repetitive butterfly stroke. We then define an $(a, b, c, d)$-repetitive butterfly stroke that is kick-to-kick temporal symmetric by imposing the equality of the Upward phase 1 and Upward phase 2 durations:

$$
t_{\mathrm{U} 1}=t_{\mathrm{U} 2}+T_{1} .
$$

Furthermore, a highly coordinated $(a, b, c, d)$-kick-to-kick temporal symmetric repetitive butterfly stroke is an $(a, b, c, d)$-kick-to-kick temporal symmetric repetitive butterfly stroke that exhibits a relatively small positive value for the normalized total delay:

$$
N T D=\sqrt{a^{2}+b^{2}+c^{2}+d^{2}}[\%] .
$$

In light of [6], a self-similar partition in highly coordinated $(a, b, c, d)$-kick-to-kick temporal symmetric repetitive butterfly strokes is presented hereafter.

Theorem 1. $\left(\mathcal{F}_{4}\right)$ : In a highly coordinated $(a, b, c, d)$-kick-to-kick temporal symmetric repetitive butterfly stroke, the sequence:

$$
\mathcal{F}_{4}:\left\{t_{\mathrm{K} 1}+t_{\mathrm{K} 2}, t_{\mathrm{U} 1}, t_{\mathrm{K} 1}+t_{\mathrm{K} 2}+t_{\mathrm{U} 1}, t_{\mathrm{S}}\right\}
$$

is a generalized Fibonacci sequence of length 4 . If $t_{\mathrm{U} 1} /\left(t_{\mathrm{K} 1}+t_{\mathrm{K} 2}\right)=\phi$, then the chain of inequalities:

$$
\frac{t_{\mathrm{K} 1}+t_{\mathrm{K} 2}+t_{\mathrm{U} 1}}{t_{\mathrm{U} 1}}=\frac{t_{\mathrm{S}}}{t_{\mathrm{K} 1}+t_{\mathrm{K} 2}+t_{\mathrm{U} 1}}=\phi
$$


holds and the sequence $\mathcal{F}_{4}$ in $(7)$ has a self-similar structure, with $t_{\mathrm{K} 1}+t_{\mathrm{K} 2}+t_{\mathrm{U} 1} \approx 61.804 \%$ of $t_{\mathrm{S}}, t_{\mathrm{U} 1} \approx 38.198 \%$ of $t_{\mathrm{S}}$ and $t_{\mathrm{K} 1}+t_{\mathrm{K} 2} \approx 23.608 \%$ of $t_{\mathrm{S}}$.

Proof. As in [6], the sequence $\mathcal{F}_{4}$ is represented through the discrete-time system:

$$
y(k+2)=y(k+1)+y(k), k=0,1,
$$

with $y(0)=t_{\mathrm{K} 1}+t_{\mathrm{K} 2}, y(1)=t_{\mathrm{U} 1}$ and $y(2)=t_{\mathrm{S}}-t_{\mathrm{U} 2}, y(3)=t_{\mathrm{S}}$. Then, it suffices to write the solution to its state-space description:

$$
\xi(k+1)=\mathcal{M} \xi(k), k=0,1
$$

as

$$
\begin{aligned}
& \xi(1)=\mathcal{M} \xi(0), \\
& \xi(2)=\mathcal{M}^{2} \xi(0),
\end{aligned}
$$

with $\xi(l)$ being the vector $[y(l), y(l+1)]^{\mathrm{T}}, l=0,1,2$, and $\mathcal{M}=[0,1 ; 1,1]$ with distinct eigenvalues $\phi, 1-\phi$ and orthogonal eigenvectors $\left(c_{\phi}=\left(1+\phi^{2}\right)^{-1 / 2}\right)$ :

$$
v_{\phi}=c_{\phi}[1, \phi]^{\mathrm{T}}, \quad v_{(1-\phi)}=c_{\phi}[\phi,-1]^{\mathrm{T}} .
$$

The following theorem characterizes a stronger self-similarity property, referred to as enhanced self-similarity. It relies on the following constraint (When $t_{\mathrm{K} 2}>t_{\mathrm{K} 1}$, it suffices to switch $t_{\mathrm{K} 1}$ and $t_{\mathrm{K} 2}$ in the $\mathcal{C}$-constraint, in the sequence of Corollary 1 (including Remark $\left(\mathcal{F}_{7}\right)$ ), in the corresponding index (24), and in the fourth comment Section 4$)$ :

$$
\mathcal{C}: t_{\mathrm{U} 1}=t_{\mathrm{K} 1}+2 t_{\mathrm{K} 2}
$$

Theorem 2. $\left(\mathcal{F}_{6}\right)$ : In a highly coordinated $(a, b, c, d)$-kick-to-kick temporal symmetric repetitive butterfly stroke under $\mathcal{C}$, the sequence (7) enforced with $\left\{t_{\mathrm{K} 1}, t_{\mathrm{K} 2}\right\}$ to the left, meaning that:

$$
\mathcal{F}_{6}:\left\{t_{\mathrm{K} 1}, t_{\mathrm{K} 2}, t_{\mathrm{K} 1}+t_{\mathrm{K} 2}, t_{\mathrm{U} 1}, t_{\mathrm{K} 1}+t_{\mathrm{K} 2}+t_{\mathrm{U} 1}, t_{\mathrm{S}}\right\},
$$

is a generalized Fibonacci sequence of length 6 . The equality $t_{\mathrm{U} 1} /\left(t_{\mathrm{K} 1}+t_{\mathrm{K} 2}\right)=\phi$ makes the sequence $\mathcal{F}_{6}$ possess an enhanced self-similar structure, with $t_{\mathrm{K} 1}+t_{\mathrm{K} 2}+t_{\mathrm{U} 1} \approx 61.804 \%$ of $t_{\mathrm{S}}$, $t_{\mathrm{U} 1} \approx 38.198 \%$ of $t_{\mathrm{S}}, t_{\mathrm{K} 1}+t_{\mathrm{K} 2} \approx 23.608 \%$ of $t_{\mathrm{S}}, t_{\mathrm{K} 2} \approx 14.591 \%$ of $t_{\mathrm{S}}, t_{\mathrm{K} 1} \approx 9.0175 \%$ of $t_{\mathrm{S}}$.

Proof. Sequence $\mathcal{F}_{6}$ can be represented by the discrete-time system:

$$
y(k+2)=y(k+1)+y(k), \quad k=0,1,2,3,
$$

with initial conditions $y(0)=t_{\mathrm{K} 1}, y(1)=t_{\mathrm{K} 2}$ and $y(2)=t_{\mathrm{K} 1}+t_{\mathrm{K} 2}, y(3)=t_{\mathrm{U} 1}, y(4)=$ $t_{\mathrm{K} 1}+t_{\mathrm{K} 2}+t_{\mathrm{U} 1}, y(5)=t_{\mathrm{S}}$. The previous analysis carried out in the proof of Theorem 1 can be extended to cover the generalized 6-length Fibonacci sequence case. In particular, the state-space representation of the system above reads:

$$
\xi(k+1)=\mathcal{M} \xi(k), \quad k=0,1,2,3,
$$

with $\xi(l)=[y(l), y(l+1)]^{\mathrm{T}}, l=0,1,2,3,4$, and $\mathcal{M}$ as in the proof of Theorem 1 . We now explicitly write: 


$$
\begin{aligned}
& {\left[\begin{array}{l}
y(2) \\
y(3)
\end{array}\right]=\phi^{2} \beta v_{\phi}+(1-\phi)^{2} \alpha v_{(1-\phi)}} \\
& {\left[\begin{array}{l}
y(3) \\
y(4)
\end{array}\right]=\phi^{3} \beta v_{\phi}+(1-\phi)^{3} \alpha v_{(1-\phi)}} \\
& {\left[\begin{array}{l}
y(4) \\
y(5)
\end{array}\right]=\phi^{4} \beta v_{\phi}+(1-\phi)^{4} \alpha v_{(1-\phi)},}
\end{aligned}
$$

in terms of:

$$
\begin{aligned}
\alpha & =\left\langle\xi(0), v_{(1-\phi)}\right\rangle=(\phi y(0)-y(1)) c_{\phi} \\
\beta & =\left\langle\xi(0), v_{\phi}\right\rangle=(y(0)+\phi y(1)) c_{\phi}=\left(1+\phi^{2}\right) c_{\phi} y(0)
\end{aligned}
$$

When $\alpha$ is equal to zero, i.e., the initial vector $\xi(0)$ has no components along the $v_{(1-\phi)^{-}}$ direction, $y(1) / y(0)=\phi$ holds and $y(2) / y(1)=\phi, y(3) / y(2)=\phi, y(4) / y(3)=\phi$, $y(5) / y(4)=\phi$ hold too.

Remark 1. $\left(\mathcal{F}_{7}\right)$ : In the very special case of $T_{1}=t_{\mathrm{K} 2}-t_{\mathrm{K} 1}$ for the highly coordinated $(a, b, c, d)$ kick-to-kick temporal symmetric repetitive butterfly stroke, the sequence (15), once enforced with $T_{1}$ to the left, becomes a generalized Fibonacci sequence of length 7 , with the equality $t_{\mathrm{U} 1} /\left(t_{\mathrm{K} 1}+t_{\mathrm{K} 2}\right)=\phi$ making the sequence $\mathcal{F}_{7}$ possess a strongly enhanced self-similar structure; $T_{1} \approx 5.5735 \%$ of $t_{\mathrm{S}}$ is additionally obtained.

\subsection{Quantitative Measures of Self-Similarity and Enhanced Self-Similarities}

Two indices $\mathcal{I}_{f, 4}, \mathcal{I}_{f, 6}$-named $\phi$-bonacci butterfly stroke number and enhanced $\phi$-bonacci butterfly stroke number - can be naturally introduced, in order to quantitatively assess selfsimilarity or enhanced self-similarities of highly coordinated $(a, b, c, d)$-kick-to-kick temporal symmetric repetitive butterfly strokes. They are, in order:

$$
\begin{aligned}
\mathcal{I}_{f, 4}= & 100\left[\left(\left(t_{\mathrm{K} 1}+t_{\mathrm{K} 2}+t_{\mathrm{U} 1}\right) / t_{\mathrm{S}}-0.62\right)^{2}+\left(t_{\mathrm{U} 1} / t_{\mathrm{S}}-0.38\right)^{2}\right. \\
& \left.+\left(\left(t_{\mathrm{K} 1}+t_{\mathrm{K} 2}\right) / t_{\mathrm{S}}-0.24\right)^{2}\right]^{1 / 2} \\
\mathcal{I}_{f, 6}= & 100\left[\left(\left(t_{\mathrm{K} 1}+t_{\mathrm{K} 2}+t_{\mathrm{U} 1}\right) / t_{\mathrm{S}}-0.62\right)^{2}+\left(t_{\mathrm{U} 1} / t_{\mathrm{S}}-0.38\right)^{2}\right. \\
& +\left(\left(t_{\mathrm{K} 1}+t_{\mathrm{K} 2}\right) / t_{\mathrm{S}}-0.24\right)^{2}+\left(t_{\mathrm{K} 2} / t_{\mathrm{S}}-0.14\right)^{2} \\
& \left.+\left(t_{\mathrm{K} 1} / t_{\mathrm{S}}-0.10\right)^{2}\right]^{1 / 2},
\end{aligned}
$$

where the values $0.62,0.38,0.24,0.14,0.10$ are used to approximate $0.61804,0.38198,0.23608$, $0.14591,0.090175$ of Theorem 1 and Theorem 2, respectively. The smaller such indices are, the stronger the self-similarity is. The just self-similarity of Theorem 1 may clearly lead to a non-zero value for $\mathcal{I}_{f, 6}$ that tends towards zero when the self-similarity tends to turn into the enhanced self-similarity.

\section{Experimental Analysis}

The feasibility of the preceding analysis is here illustrated by the dedicated analysis of butterfly stroke training sessions for: (i) seven international-level swimmers, namely IL1 (male, $31 \mathrm{y}, 190 \mathrm{~cm}, 80 \mathrm{~kg}$ ), IL2 (female, $31 \mathrm{y}, 170 \mathrm{~cm}, 65 \mathrm{~kg}$ ), IL3 (female, $27 \mathrm{y}, 168 \mathrm{~cm}, 58 \mathrm{~kg}$ ), IL4 (male, $20 \mathrm{y}, 196 \mathrm{~cm}, 80 \mathrm{~kg}$ ), IL5 (male, $19 \mathrm{y}, 180 \mathrm{~cm}, 73 \mathrm{~kg}$ ), IL6 (male, $19 \mathrm{y}, 193 \mathrm{~cm}, 85 \mathrm{~kg}$ ), IL7 (female, $27 \mathrm{y}, 173 \mathrm{~cm}, 66 \mathrm{~kg}$ ); (ii) two national-level swimmers, namely, NL1 (female, $19 \mathrm{y}, 166 \mathrm{~cm}, 55 \mathrm{~kg}$ ), NL2 (male, $25 \mathrm{y}, 185 \mathrm{~cm}, 95 \mathrm{~kg}$ ), all of them swimming at their own middle distance pace. In particular, the above international-level swimmers and nationallevel swimmers are reported, within the corresponding sets, in order of physical shape 
(measured as race performance capabilities) at the moment of data acquisition. While the international-level swimmers IL1-IL7 compete at major international events on a regular basis and hold national/international records, the national-level swimmers NL1-NL2 are national medalists who compete on a regular basis of major national events (with NL1 being close to becoming an international-level swimmer). The analysis was performed by using high frame rate videos (100 for IL1, NL2; 120 fps for IL2-IL7, NL1) of stable strokes via the 2D BioMovie ERGO system at http:/ / www.infolabmedia.eu/ (accessed on 7 June 2021).

\subsection{Phase Durations and Interlimb Coordination}

Phase and delay durations for all the swimmers IL1-IL7, NL1-NL2 are reported in Tables 1-4: $(a, b, c, d)$-(almost) kick-to-kick temporal symmetric repetitive butterfly strokes $\mathrm{S}$ (under constraints $\mathcal{C}$ ) are exhibited (Kick-to-kick temporal symmetry appears to be almost verified for IL1-IL7, NL1-NL2, with the modulus of the difference between $t_{\mathrm{U} 1}$ and $t_{\mathrm{U} 2}+T_{1}$ belonging to the set $[5,67] \mathrm{ms}$. Constraint $\mathcal{C}$ even appears to be almost verified for IL1-IL7, NL1-NL2, with the modulus of the difference between $t_{\mathrm{U} 1}$ and $t_{\mathrm{K} 1}+2 t_{\mathrm{K} 2}$ belonging to the set $[1,81] \mathrm{ms}$.), characterized by the $(a, b, c, d)$ - and NTD-values reported in Tables 3 and 4 . In accordance with the evidence of [10] on elite swimmers, all of such relatively small NTDs define highly coordinated strokes. All nine swimmers' strokes exhibit a negative delay $T_{2}$ (leading to a lag time in glide position) and a positive delay $T_{3}$. On the other hand, in contrast to the international-level swimmers IL1, IL3-IL6, the international-level swimmers IL2, IL7 and the national-level swimmers NL1, NL2 are characterized by a small negative superposition of two contradictory actions $\left(T_{1}<0\right)$. Furthermore, $T_{4}=0$ for NL1, whereas $T_{4}$ is negative for IL1-IL7 and NL2.

Table 1. Experimental data for swimmers IL1-IL7 and NL1-NL2: arm phase durations (in seconds).

\begin{tabular}{cccccc}
\hline & $\boldsymbol{t}_{\mathrm{EC}}$ & $\boldsymbol{t}_{\text {PL }}$ & $\boldsymbol{t}_{\text {PS }}$ & $\boldsymbol{t}_{\text {RE }}$ & $\boldsymbol{t}_{\mathbf{S}}$ \\
\hline IL1 & 0.220 & 0.220 & 0.270 & 0.440 & 1.150 \\
IL2 & 0.233 & 0.183 & 0.316 & 0.333 & 1.065 \\
IL3 & 0.308 & 0.158 & 0.266 & 0.333 & 1.065 \\
IL4 & 0.375 & 0.200 & 0.258 & 0.407 & 1.240 \\
IL5 & 0.291 & 0.291 & 0.250 & 0.400 & 1.232 \\
IL6 & 0.300 & 0.283 & 0.333 & 0.367 & 1.283 \\
IL7 & 0.234 & 0.175 & 0.358 & 0.417 & 1.184 \\
\hline NL1 & 0.266 & 0.166 & 0.308 & 0.326 & 1.066 \\
NL2 & 0.220 & 0.150 & 0.200 & 0.330 & 0.900 \\
\hline
\end{tabular}

Table 2. Experimental data for swimmers IL1-IL7 and NL1-NL2: leg phase durations (in seconds).

\begin{tabular}{cccccc}
\hline & $\boldsymbol{t}_{\mathbf{K} \mathbf{1}}$ & $\boldsymbol{t}_{\mathbf{K} \mathbf{2}}$ & $\boldsymbol{t}_{\mathrm{U} \mathbf{1}}$ & $\boldsymbol{t}_{\mathbf{U} \mathbf{2}}$ & $\boldsymbol{t}_{\mathbf{S}}$ \\
\hline IL1 & 0.120 & 0.160 & 0.420 & 0.450 & 1.150 \\
IL2 & 0.108 & 0.166 & 0.400 & 0.466 & 1.065 \\
IL3 & 0.125 & 0.150 & 0.391 & 0.383 & 1.065 \\
IL4 & 0.133 & 0.191 & 0.441 & 0.450 & 1.240 \\
IL5 & 0.120 & 0.141 & 0.483 & 0.430 & 1.232 \\
IL6 & 0.150 & 0.166 & 0.517 & 0.417 & 1.283 \\
IL7 & 0.150 & 0.158 & 0.467 & 0.475 & 1.184 \\
\hline NL1 & 0.108 & 0.183 & 0.417 & 0.408 & 1.066 \\
NL2 & 0.120 & 0.140 & 0.330 & 0.330 & 0.900 \\
\hline
\end{tabular}


Table 3. Delays and related values for swimmers IL1-IL7 and NL1-NL2: delay durations (in seconds) and NTD.

\begin{tabular}{crcccc}
\hline & $\boldsymbol{T}_{\mathbf{1}}$ & $\boldsymbol{T}_{\mathbf{2}}$ & $\boldsymbol{T}_{\mathbf{3}}$ & $\boldsymbol{T}_{\mathbf{4}}$ & NTD \\
\hline IL1 & 0.000 & -0.100 & 0.100 & -0.010 & $12.320 \%$ \\
IL2 & -0.075 & -0.200 & 0.017 & -0.133 & $23.680 \%$ \\
IL3 & 0.016 & -0.167 & 0.066 & -0.050 & $17.570 \%$ \\
IL4 & 0.025 & -0.217 & 0.024 & -0.043 & $18.060 \%$ \\
IL5 & 0.058 & -0.113 & 0.079 & -0.030 & $12.380 \%$ \\
IL6 & 0.033 & -0.117 & 0.117 & -0.050 & $13.720 \%$ \\
IL7 & -0.066 & -0.150 & 0.142 & -0.058 & $18.960 \%$ \\
\hline NL1 & -0.050 & -0.208 & 0.043 & -0.082 & $21.860 \%$ \\
NL2 & -0.020 & -0.120 & 0.060 & 0.000 & $15.070 \%$ \\
\hline
\end{tabular}

Table 4. Delays and related values for swimmers IL1-IL7 and NL1-NL2: $(a, b, c, d)$-values.

\begin{tabular}{crccc}
\hline & $\boldsymbol{a}$ & $\boldsymbol{b}$ & $\boldsymbol{c}$ & $\boldsymbol{d}$ \\
\hline IL1 & $0.00 \%$ & $-8.69 \%$ & $8.69 \%$ & $-0.87 \%$ \\
IL2 & $-7.04 \%$ & $-18.78 \%$ & $1.60 \%$ & $-12.49 \%$ \\
IL3 & $1.50 \%$ & $-15.68 \%$ & $6.20 \%$ & $-4.69 \%$ \\
IL4 & $2.02 \%$ & $-17.50 \%$ & $1.94 \%$ & $-3.47 \%$ \\
IL5 & $4.71 \%$ & $-9.17 \%$ & $6.41 \%$ & $-2.44 \%$ \\
IL6 & $2.57 \%$ & $-9.12 \%$ & $9.12 \%$ & $-3.90 \%$ \\
IL7 & $-5.57 \%$ & $-12.67 \%$ & $11.99 \%$ & $-4.90 \%$ \\
\hline NL1 & $-4.69 \%$ & $-19.51 \%$ & $4.03 \%$ & $-7.69 \%$ \\
NL2 & $-2.22 \%$ & $-13.33 \%$ & $6.67 \%$ & $0.00 \%$ \\
\hline
\end{tabular}

\subsection{Self-Similarity Analysis}

The aggregate phase percentage values for the international-level swimmers IL1-IL7 and the national-level swimmers NL1-NL2 are reported in Tables 5 and 6, along with the values for the indices $\mathcal{I}_{f, 4}$ and $\mathcal{I}_{f, 6}$ in (23) and (24). Comments are in order:

- $\quad$ Rather small values are obtained for IL1-IL7, with IL1's one being the smallest, owing to the strict closeness of the corresponding phase percentage values to $62 \%, 38 \%, 24 \%$, $14 \%$, and $10 \%$;

- Relatively large reductions in the self-similarity and enhanced self-similarity magnitudes (especially of the latter) appear for the national-level swimmers NL1-NL2 when compared to the international-level swimmers IL1-IL7;

- The $\mathcal{I}_{f, 4^{-}}$and $\mathcal{I}_{f, 6^{-}}$values turn out to reproduce the order of physical shape within the two swimmers' set;

- IL5 even presents an $a=T_{1} / t_{\mathrm{S}}$-value (4.71\%) that is close to the one (5.57\%) characterizing the strongly enhanced self-similar structure of Remark $\left(\mathcal{F}_{7}\right)$;

- The slight differences in the phase durations of Tables 1 and 2 (compare, for instance, IL2 to NL1, or IL4 to IL5, or IL2 to IL3), which lead to the differences in self-similarity magnitudes of Tables 5 and 6, have been successfully identified via the high frame rate analysis used in this paper, with the self-similarity information complementing the delay partition values of Tables 3 and 4;

- Larger percentage reductions in enhanced self-similarity (with respect to self-similarity) are exhibited by IL2, IL3, IL5-when compared to IL1, IL4, IL5, IL6, NL1, NL2—so that the $\mathcal{I}_{f, 6^{-}}$values for IL2-IL3 and IL5 tend to thicken (more than the $\mathcal{I}_{f, 4}$-ones) towards the $\mathcal{I}_{f, 6^{-}}$values for IL4 and IL6, respectively. 
Table 5. Data analysis for swimmers IL1-IL7 and NL1-NL2: aggregate phases (percentage values).

\begin{tabular}{cccccc}
\hline & $\left(t_{\mathrm{K} 1}+t_{\mathrm{K} 2}+t_{\mathrm{U} 1}\right) / t_{\mathrm{S}}$ & $t_{\mathrm{U} 1} / t_{\mathrm{S}}$ & $\left(t_{\mathrm{K} 1}+t_{\mathrm{K} 2}\right) / t_{\mathrm{S}}$ & $t_{\mathrm{K} 2} / t_{\mathrm{S}}$ & $t_{\mathrm{K} 1} / t_{\mathrm{S}}$ \\
\hline IL1 & 60.87 & 36.52 & 24.35 & 13.91 & 10.43 \\
IL2 & 63.29 & 37.56 & 25.73 & 15.59 & 10.14 \\
IL3 & 62.54 & 36.71 & 25.82 & 14.08 & 11.74 \\
IL4 & 61.69 & 35.56 & 26.13 & 15.40 & 10.73 \\
IL5 & 60.39 & 39.20 & 21.19 & 11.44 & 9.74 \\
IL6 & 64.93 & 40.30 & 24.63 & 12.94 & 11.69 \\
IL7 & 65.46 & 39.44 & 26.01 & 13.34 & 12.67 \\
\hline NL1 & 66.42 & 39.12 & 27.30 & 17.17 & 10.13 \\
NL2 & 65.56 & 36.67 & 28.89 & 15.56 & 13.33 \\
\hline
\end{tabular}

Table 6. Data analysis for swimmers IL1-IL7 and NL1-NL2: values of indices (23) and (24).

\begin{tabular}{ccc}
\hline & $\mathcal{I}_{f, 4}$ & $\mathcal{I}_{f, \mathbf{6}}$ \\
\hline IL1 & 1.89 & 1.94 \\
IL2 & 2.20 & 2.72 \\
IL3 & 2.30 & 2.88 \\
IL4 & 3.25 & 3.61 \\
IL5 & 3.45 & 4.31 \\
IL6 & 3.78 & 4.27 \\
IL7 & 4.25 & 5.06 \\
\hline NL1 & 5.63 & 6.46 \\
NL2 & 6.19 & 7.20 \\
\hline
\end{tabular}

\section{Discussion}

By following up the direction highlighting the connections of human walking and running gaits and front crawl swimming strokes with the generalized Fibonacci sequences and golden ratio, this paper aimed to provide an answer to the question regarding the existence of subtle and unveiled self-similar structures at the root of the butterfly stroke of elite swimmers, for whom —as in dolphins and fish [18] — energy accrued by raising the upper body is transmitted along the body through wave-like movements [19]. Harmonically characterizing butterfly strokes through self-similarity-in both its simple and enhanced versions-and the $\phi$-bonacci butterfly stroke numbers constitutes the original contribution of this paper, as a relevant application of computational number theory involving generalized Fibonacci sequences.

When compared to the front crawl scenario of [6] (concerning the arm stroke):

- Theorem 1 and Theorem 2 provide partition constraints that regard the leg stroke, with the inter-delay composition linking the arm phase partition with the leg phase partition;

- $\quad$ The four-length generalized Fibonacci sequence $\mathcal{F}_{4}$ of Theorem 1 is generated by $t_{\mathrm{K} 1}+t_{\mathrm{K} 2}$, which plays the role of the recovery phase duration in the front crawl of [6] and of the double support (double float) duration in walking (running) of [2];

- The durations $t_{\mathrm{U} 1}$ and $t_{\mathrm{U} 2}+T_{1}$ play the role of $t_{\mathcal{F}_{a}}$ and $t_{\mathcal{F}_{b}}$ in the front crawl stroke in [6] and of the right and left swing (right and left stance) durations in the asymmetric walking (running) of [2,5], with the involved equality between the durations of such phases simply transposing the swing (stance)-symmetry constraint of symmetric walking (running);

- In light of the twelve-tone equal temperament-based interpretation, constraint $\mathcal{C}$ imposes that the stroke duration $t_{\mathrm{S}}$ of an $(a, b, c, d)$-kick-to-kick temporal symmetric repetitive butterfly stroke equals the sum of the durations of eight disjoint sub-phases, three among them with duration $t_{\mathrm{K} 1}$ and five among them with duration $t_{\mathrm{K} 2}$ : according 
to [6], notes D4, E4, G4, C5 correspond, in the (suspended and restored) C-variant Cadd2-chord, to the frequencies: $f_{3}(\mathrm{D} 4)=f_{0} 2^{\frac{3}{12}}=293.7 \mathrm{~Hz}, f_{5}(\mathrm{E} 4)=f_{0} 2^{\frac{5}{12}}=$ $329.6 \mathrm{~Hz}, f_{8}(\mathrm{G} 4)=f_{0} 2^{\frac{8}{12}}=391.96 \mathrm{~Hz}, f_{13}(\mathrm{C} 5)=f_{0} 2^{1 \frac{13}{12}}=2 f_{1}(\mathrm{C} 4)=523.2 \mathrm{~Hz}$ $\left(f_{0}=246.9175 \mathrm{~Hz}\right)$, with $n=3,5,8,13$ corresponding to elements of a Fibonacci sequence and with the ratios $5 / 3 \approx 1.667,8 / 5=1.6,13 / 8=1.625$ of consecutive elements of such sequence quickly getting close to $\phi$;

- The above results-again occurring in elite swimmers-confirm that, in contrast to walking, a precise swimming technique (coming from a considerable amount of practice and instruction repeating specifically precise movements for a long enough time, while producing rhythmic motor patterns through the interrelationship between cortical input, central pattern generator (CPG), and sensory feedback) is relevantly involved (the intra-cyclic variation of the horizontal velocity of the hip is larger in non-expert swimmers than their expert counterparts [20]) as recalled by [6], the fractal dimension is $1.8-1.9$ for highly qualified expert swimmers [20], whereas it is 1.1-1.4 for on-land walking [21].

\section{Practical Implications}

Self-similarity, as defined in this paper, may become a reference point in the formulation of advanced training programs (on the basis of (23), (24)) for elite swimmers, while: (i) going beyond the evaluation of the inter-limb synchronicity level, classically used to assess the performance of high-level butterfly stroke swimmers; and (ii) not affecting the peculiar stroke duration of the swimmer. It is clear that improvements of the muscle strength remain valid as well, but at a different level of analysis. The findings of this paper might also provide comparative (quantitative) information about: (i) the physical recovery level of the swimmer after a hard and intense workout; (ii) the conditioning of the rhythmic neural patterns of the swimmer during the contingent-moment-based swimming actions; and (iii) the swimmers' improvements over time and the necessary correction actions to improve the performance. Indeed, at a high-level, at which inter-individual coordination variability reduces to a narrow range of movement variants [20], each small change that a practitioner may perform might lead to a relevant change in the dynamic interaction of the systems components [22].

\section{Strengths and Limits}

The derivations of this paper seem to provide meaningful information about both the swimmer's level and physical shape, which complement the analysis of the normalized total delay. The results of this paper also seek to open new research directions aiming to connect computational number theory (involving generalized Fibonacci sequence) to human neural system modulating principles at the root of highly effective cyclic rhythmic patterns of swimming. Anyway, the results of the present study should be interpreted with caution, owing to its specific limits, such as: (i) the small size of the samples enrolled in the experiments; and (ii) the dependence on high-frame rate analyses. Many aspects should be further investigated in future studies. One of them relies on the fact that the present analysis is focused on the proportions among the stroke phases, limiting this type of analysis to temporal features. Furthermore, it could be certainly interesting to put self-similarity in relationship with the role played by different sensory information in constructing and maintaining the aforementioned stroke harmony.

\section{Forecast}

It is worth noticing how Figure 3 of [23] (200 m-pace, high-level male swimmer) seems to respect such a self-similar partition in breaststroke, with the arm recovery and the out-sweep phases in place of $t_{\mathcal{F}_{a}}, t_{\mathcal{F}_{b}}$ and the arm in-sweep phase in place of the front crawl stroke recovery phase. On the other hand, we recognized the same self-similar partition in the fourth stroke of the start phase (which accounts for a greater percentage of the race success [24]) in elite sprint kayaking contests, once the sequence generated 
by $\min \left\{t_{\mathrm{Ext}}, t_{\mathrm{Pull}}\right\}, t_{\mathrm{Imm}}+\max \left\{t_{\mathrm{Ext}}, t_{\mathrm{Pull}}\right\}$ is considered under the experimentally verified constraint $t_{\mathrm{Rec}}-\left(2 t_{\mathrm{Imm}}+t_{\mathrm{Pull}}+t_{\mathrm{Ext}}+\max \left\{t_{\mathrm{Ext}}, t_{\mathrm{Pull}}\right\}\right)$, in terms of the paddle immersion, pull, paddle extraction and recovery phases of the stroke. Analogously, the reader can appreciate in Table 2 and Figure 2 of [25] how, in the forward swing of the forehand tennis stroke (cross court and down the line situations) of elite tennis players, the same self-similar partition can be found owing to: (i) a temporal distance between the instants of maximum racquet rotation angle and maximum hip linear velocity that is almost equal to the temporal distance between the instants of maximum trunk angular velocity and ball impact; (ii) instants of maximum wrist linear velocity and maximum trunk angular velocity that occur at about $24 \%$ and $38 \%$ of the temporal distance duration between the ball impact and the maximum racquet rotation angle, with these proportions not being exhibited by high-level tennis players. All of these scenarios seem to suggest that harmonic properties in top-level athletes - possessing talent, natural aptitudes or skills- are an expression of a harmonic rhythm generated by CPG, in which the repetitive proportions of phases act as an attractor for a smooth, efficient, graceful and melodic flow of movements in which different limbs, joints and muscles are controlled at the same time and with a high level of coordination.

\section{Conclusions}

A temporal partition for the butterfly stroke that is harmonically characterized by Fibonacci sequence-based self-similarity - in both its simple and enhanced versions-was presented and discussed. High frame rate video-based experiments on nine elite swimmers (seven of international level and two of national level) were included. The practical implications, strengths and limits of the analysis were discussed.

Author Contributions: Conceptualization: C.M.V., I.F., V.B., G.A.; Methodology: C.M.V., V.B., G.A., R.J.; Software and Resources: C.M.V., V.B., C.R., I.F.; Formal Analysis: C.M.V.; Validation, Investigation: C.M.V., I.F., V.B., C.R., G.A.; Writing-Original Draft: C.M.V.; Writing—Review and Editing: C.M.V., V.B., R.J., G.A. All authors have read and agreed to the published version of the manuscript.

Funding: This research received no external funding.

Institutional Review Board Statement: The Internal Research Board (University of Rome Tor Vergata) examined the documentation about this paper and recognized that: it is in accordance with ethical principles for medical research involving human subjects of Declaration of Helsinki; it just assesses motor skills during standard practice routines, while involving no invasive technique.

Informed Consent Statement: Informed consent was obtained from all subjects involved in the study.

Data Availability Statement: No publicly archived datasets were analyzed or generated during the study.

Acknowledgments: The first author is indebted to Marcello Molinari, Technical Director of "New Country Club Frascati" and "Federazione Italiana Tennis (FIT)"- National Master, for drawing attention to the phase partitions of the forehand tennis stroke.

Conflicts of Interest: The authors declare no conflict of interest.

\section{References}

1. Chen, L.; Wang, X. The power sums involving fibonacci polynomials and their applications. Symmetry 2019, 11, 635. [CrossRef]

2. Verrelli, C.M.; Iosa, M.; Roselli, P.; Pisani, A.; Giannini, F.; Saggio, G. Generalized finite-length Fibonacci sequences in healthy and pathological human walking: Gait recursivity, asymmetry, consistency, self-similarity, and variability. In Frontiers in Human Neuroscience; Special Issue: Rhythmic Patterns in Neuroscience and Human Physiology; to be published .

3. Horadam, A.F. A generalized Fibonacci sequence. Am. Math. Mon. 1961, 68, 455-459. [CrossRef]

4. Iosa, M.; Fusco, A.; Marchetti, F.; Morone, G.; Caltagirone, C.; Paolucci, S.; Peppe, A. The golden ratio of gait harmony: Repetitive proportions of repetitive gait phases. Biomed Res. Int. 2013, 2013, 918642 . [CrossRef]

5. Marino, R.; Verrelli, C.M.; Gnucci, M. Synchronicity rectangle for temporal gait analysis: Application to Parkinson's Disease. Biomed. Signal Process. Control 2020, 62, 102156. [CrossRef]

6. Verrelli, C.M.; Romagnoli, C.; Jackson, R.R.; Ferretti, I.; Annino, G.; Bonaiuto, V. Front crawl stroke in swimming: Ratios of phase durations and self-similarity. J. Biomech. 2021, 118, 110267. [CrossRef] [PubMed] 
7. Taylor, G.K.; Nudds, R.L.; Thomas, A.L.R. Flying and swimming animals cruise at a Strouhal numer tuned for high power efficiency. Lett. Nat. 2003, 425, 707-711. [CrossRef] [PubMed]

8. Eloy, C. Optimal Strouhal number for swimming animals. J. Fluids Struct. 2012, 30, 215-218. [CrossRef]

9. Barbosa, T.M.; Keskinen, K.L.; Fernandes, R.; Colaço, P.; Lima, A.B.; Vilas-Boas, J.P. Energy cost and intracyclic variation of the velocity of the centre of mass in butterfly stroke. Eur. J. Appl. Physiol. 2005, 93, 519-523. [CrossRef]

10. Seifert, L.; Boulesteix, L.; Chollet, D.; Vilas-Boas, J.P. Differences in spatial-temporal parameters and arm-leg coordination in butterfly stroke as a function of race pace, skill and gender. Hum. Mov. Sci. 2008, 27, 96-111. [CrossRef]

11. Di Prampero, P.E.; Pendergast, D.R.; Wilson, D.W.; Rennie, D.W. Blood Lactic Acid Concentrations in High Velocity Swimming. In Swimming Medicine IV, Proceedings of the Fourth International Congress on Swimming Medicine, Stockholm, Sweden; Eriksson, B., Furberg, B., Eds.; University Park Press: Baltimore, MD, USA, 1978; pp. 249-261.

12. Chollet, D.; Seifert, L.; Boulesteix, L.; Carter, M. Arm-leg coordination in elite butterfly swimmers. Int. J. Sport Med. 2006, 27, 322-329. [CrossRef]

13. Seifert, L.; Delignieres, D.; Chollet, D. Effect of expertise on butterfly stroke coordination. J. Sport. Sci. 2007, 25, 131-141. [CrossRef] [PubMed]

14. Lanotte, N.; Annino, G.; Bifaretti, S.; Gatta, G.; Romagnoli, C.; Salvucci, A.; Bonaiuto, V. A new device for propulsion analysis in swimming. Proceedings 2018, 2, 285. [CrossRef]

15. Tourny-Chollet, C.; Chollet, D.; Hogie, S.; Papparodopoulos, C. Kinematic analysis of butterfly turns of international and national swimmers. J. Sport. Sci. 2002, 20, 383-390. [CrossRef]

16. Mason, B.R.; Tong, Z.; Richards, R.J. A biomechanical analysis of the butterfly stroke. Excel 1991, 7, 6-11.

17. Chollet, D.; Chalies, S.; Chatard, J.C. A new index of coordination for the crawl: Description and usefulness. Int. J. Sport. Med. 1999, 21, 54-59. [CrossRef] [PubMed]

18. Ungerechts, B.E. A comparison of the movements of the rear parts of dolphins and butterfly swimmers. In Biomechanics and Medicine in Swimming; Hollander, A.P., Huijing, P.A., de Groot, G. , Eds.; Human Kinetics: Champaign, IL, USA, 1983 ; pp. $215-221$.

19. Sanders, R.H.; Cappaert, J.M.; Devlin, R.K. Wave Characteristics of butterfly swimming. J. Biomech. 1995, 28, 9-16. [CrossRef]

20. Barbosa, T.M.; Goh, W.X.; Morais, J.E.; Costa, M.J. Variation of linear and nonlinear parameters in the swim strokes according to the level of expertise. Mot. Control 2017, 21, 312-326. [CrossRef]

21. Schiffman, J.M.; Chelidze, D.; Adams, A.; Segala, D.B.; Hasselquist, L. Nonlinear analysis of gait kinematics to track changes in oxygen consumption in prolonged load carriage walking: A pilot study. J. Biomech. 2009, 42, 2196-2199. [CrossRef] [PubMed]

22. Barbosa, T.M.; Goh, W.X.; Morais, J.E.; Costa, M.J. Comparison of classical kinematics, entropy, and fractal properties as measures of complexity of the motor system in swimming. Front. Psychol. 2016, 7, 1-7. [CrossRef]

23. Takagi, H.; Sugimoto, S.; Nishijima, N.; Wilson, B. Differences in stroke phases, arm-leg coordination and velocity fluctuation due to event, gender and performance level in breaststroke. Sport. Biomech. 2004, 3, 15-27. [CrossRef] [PubMed]

24. Ualí, I.; Herrero, A.J.; Garatachea, N.; Marín, P.J.; Alvear-Ordenes, I.; García-López, D. Maximal strength on different resistance training rowing exercises predicts start phase performance in elite kayakers. J. Strenght Cond. Res. 2012, 26, 941-946. [CrossRef] [PubMed]

25. Landlinger, J.; Lindinger, S.; Stöggl, T.; Wagner, H.; Müller, E. Key factors and timing patterns in the tennis forehand of different skill levels. J. Sport. Sci. Med. 2010, 9, 643-651. 